\title{
The National Plant Diagnostic Network: Partnering to Protect Plant Systems
}

\author{
James P. Stack \\ Department of Plant Pathology, Kansas State University, Manhattan \\ Richard M. Bostock \\ Department of Plant Pathology, University of California, Davis
}

Raymond Hammerschmidt

Department of Plant, Soil and Microbial Sciences, Michigan State University, East Lansing

Jeffrey B. Jones

Department of Plant Pathology, University of Florida, Gainesville

\author{
Eileen Luke \\ Center for Environmental and Regulatory Information Systems, Purdue University, \\ West Lafayette, Indiana
}

The National Plant Diagnostic Network (NPDN) has developed into a critical component of the plant biosecurity infrastructure of the United States (16). The vision set forth in 2002 for a distributed but coordinated system of plant diagnostic laboratories at land grant universities and state departments of agriculture has been realized $(4,13,15)$. NPDN, in concept and in practice, has become a model for cooperation among the public and private entities necessary to protect our natural and agricultural plant resources. Aggregated into five regional networks, NPDN laboratories upload diagnostic data records into a National Data Repository at Purdue University (Fig. 1). The diagnostic data records comprise 18 fields including the common and scientific names of the host, pathogen, and pest, relevant dates, identification numbers for the sample, submitter, and submitting laboratory, and the county of origin of the sample. By facilitating early detection and providing triage and surge support during plant disease outbreaks and arthropod pest infestations, NPDN has become an important partner among federal, state, and local plant protection agencies and with the industries that support plant protection. In addition to plant pathogens and plant-associated arthropods, many NPDN laboratories also identify native and invasive plants. The majority of effort in NPDN labs revolves around plant pathogens and arthropods; however, plant diagnosticians routinely process a broad diversity of samples, including edible and poisonous fungi, potentially venomous spiders, aquatic algae, and molds in buildings. Although there is still much to do, a solid foundation has been laid upon which to build a sustainable capability to assure the early detection of plant disease outbreaks and arthropod pest infestations, the accurate diagnoses of high consequence pathogens and pests, and the timely and secure communication of information relevant to rapid response and effective mitigation. It is this last task, that of timely and secure communications, that often generates misunderstandings among partners in plant protection and is the primary subject of this paper.

\section{Responsible Communication of Accurate Information}

The detection and identification of a new plant disease or pest in either managed (e.g., agricultural production field, horticultural

Corresponding author: James P. Stack, E-mail: jstack@ksu.edu

http://dx.doi.org/10.1094/PDIS-08-13-0876-FE

(C) 2014 The American Phytopathological Society greenhouse, or nursery) or natural (e.g., forest, prairie) plant systems can have significant economic and ecological ramifications. The disease, the pest, and the system affected determine whether such detections trigger an array of response and mitigation measures that include the implementation of active or passive surveillance programs, eradication and quarantine protocols, or the application of preventative and curative chemicals. Each of these practices requires an investment of human, physical, and financial resources, the cost of which becomes part of the total impact resulting from the introduction. Because response actions and mitigation measures are often specific to the introduced pathogen or pest and the systems affected, an accurate diagnosis is essential to ensure that the actions taken will be effective. Similarly, any information regarding new introductions and outbreaks must be accurate and be communicated in a timely manner: accurate so that the response actions that are taken are appropriate to the introduced pathogen or pest and timely so that the response actions are taken in a timeframe to optimize effectiveness. Response and mitigation measures can have impacts beyond the target pathogen or pest. For example, the application of chemical management (e.g., fungicides, insecticides) to prevent the establishment and spread of an introduced pathogen or pest may have nontarget effects on beneficial organisms; best management practices promote the application of chemicals only when and where necessary. Eradication measures often include the destruction of plants within a specified radius of the detection site. This is true whether the plants are directly affected by the introduced organism or not. One nontarget effect of plant eradication is the destruction of habitat and food supply for wildlife. It is important to implement mitigation measures only when and where necessary to prevent unintended economic, ecological, and environmental impacts. Whether a sample is positive or negative for a high consequence pathogen or pest, either a correct or incorrect diagnosis can result in significant impacts (Inset 1).

Generating accurate information. NPDN diagnostic laboratories are staffed with highly skilled and experienced professionals who readily share their expertise across NPDN. Easy access to this vast array of expertise has been extremely valuable to new diagnosticians just beginning their careers. NPDN also provides opportunities for diagnosticians to enhance their capabilities through advanced training workshops on technologies, pathogens, and pests. U.S. Department of Agriculture (USDA) Animal and Plant Health Inspection Service (APHIS) Plant Protection and Quarantine 
(PPQ) Center for Plant Health Science and Technology (CPHST) offers to NPDN diagnosticians high quality workshops for regulatory pathogens and pests. NPDN has begun the development of a laboratory accreditation system for NPDN diagnostic laboratories. The objective of the System for True Accurate and Reliable Diagnostics (STAR-D) accreditation program will be to enhance the credibility of NPDN diagnoses by promoting a set of standards that assures high quality diagnostics and well-functioning Network laboratories. The NPDN Lab Accreditation program is not intended to supersede existing lab accreditation systems that may already be in place; e.g., an NPDN-affiliated state department of agriculture diagnostic lab may have an independent accreditation program. In partnership with USDA APHIS PPQ CPHST, many NPDN diagnosticians participate in a provisional protocol certification system for certain high consequence organisms. This protocol certification system involves annual proficiency testing for protocol execution and interpretation of results by diagnosticians. All of these programs contribute to the NPDN goal of high quality diagnostics in all NPDN laboratories.

NPDN has also provided a platform to facilitate collaboration among diagnostic laboratories at universities and governmental agencies. Five NPDN diagnostic labs collaborated with the USDA Agricultural Research Service lab at Fort Detrick to test the robustness of a newly developed diagnostic protocol for the Asian soybean rust pathogen (7). An NPDN Regional Center diagnostic laboratory collaborated with USDA APHIS PPQ CPHST scientists to determine the accuracy and dependability of several diagnostic technologies for Phytophthora ramorum (3). NPDN has become a trusted member of the diagnostic community.

Information security. An effective response to any emergency, including plant disease outbreaks and arthropod pest infestations, is often dependent upon the timely communication of accurate information. There is great value in the commerce of plants and plant products. In agricultural environments, information about disease outbreaks and arthropod pest infestations can result in decisions to stop the transportation and sale of plants and plant products resulting in enormous negative economic impacts to individuals and industries. Consequently, information regarding diseases and pests in plants and plant products is very sensitive, and secure, effective communication protocols are necessary to protect plant systems and the businesses that support those systems. Incomplete or inaccurate information can result in negative consequences or impacts (8). Critical communications among NPDN laboratories regarding unknown samples or suspected regulatory samples are accomplished by telephone, e-mail, or through a web-based encrypted communications system depending upon the sensitivity of the information (14). The NPDN Repository of Diagnostic Data is behind a password-protected, encrypted web-based system to restrict access to diagnostic records.

Responsible communication. When a potentially new pathogen or pest has been detected, the temptation may be to communicate as much information as possible, as quickly as possible, in order to facilitate preparedness, even when the diagnosis of that new pathogen or pest is preliminary, e.g., a presumptive positive or even an unknown. From a preparedness standpoint, this appears to be logical; to inform those who may be impacted. However, significant unintended consequences may result from premature communications, including severe adverse economic impacts, the unnecessary implementation of environmentally costly mitigation measures, and impairment of the regulatory measures necessary to protect an entire industry (Inset 1). At times, it may appear that there is a dilemma between protecting individual producers, protecting plants and plant systems, and protecting plant trade, e.g., protecting a wheat field versus protecting the wheat industry or protecting a nursery operation versus protecting the nursery industry. This apparent dilemma can become a source of disagreement and conflict among plant protection professionals who have distinct but overlapping missions, e.g., a land grant university extension specialist and a state or federal regulatory official. This is the very reason why effective and trusted communications are essential in every state and every organization (6).

\section{NPDN Mission}

Whether an outbreak is the result of a newly introduced, a recurring, or an emerging pathogen or pest, early detection, accurate diagnosis, and rapid response are essential to minimize impacts and to reduce the probability of establishment and spread of the potentially damaging organism. NPDN, state departments of agriculture, USDA APHIS PPQ, and the industries that support plant systems have independent but overlapping roles and responsibilities in the shared mission of protecting plant health (Fig. 2). The mission of NPDN is to provide a cohesive, distributed system to quickly detect and identify pests and pathogens of concern to plant systems and to communicate that information to appropriate re-

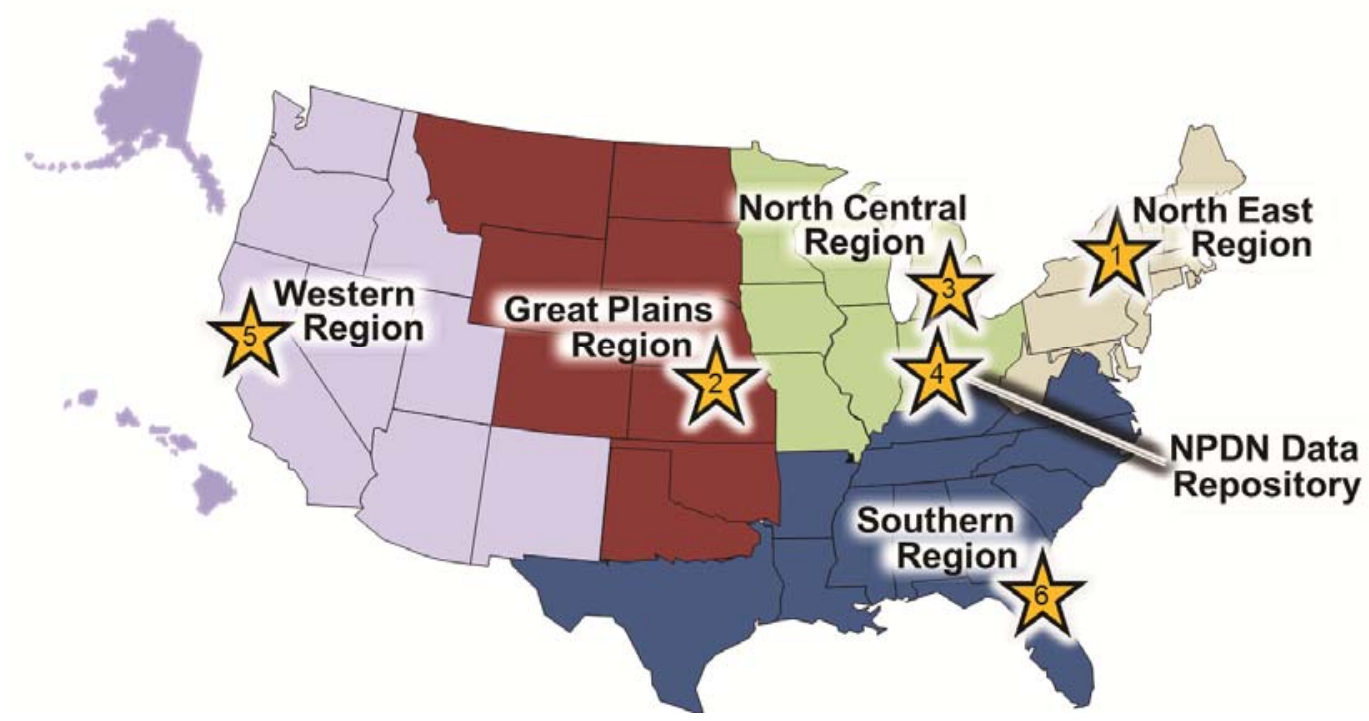

Fig. 1. The National Plant Diagnostic Network (NPDN) is comprised of five regional networks and a national data repository. Each regional network has a regional center to provide administrative management, program coordination, and diagnostic support. NPDN Regional Centers are located at Cornell University (Northeast Plant Diagnostic Network; www.nepdn.org), Kansas State University (Great Plains Diagnostic Network; www.gpdn.org), Michigan State University (North Central Plant Diagnostic Network; www.ncpdn.org), Purdue University (NPDN Data Repository; https://npdn.ceris.purdue.edu/), the University of California/Davis (Western Plant Diagnostic Network; www.wpdn.org), and the University of Florida (Southern Plant Diagnostic Network; www.spdn.org). Location and contact information for individual state diagnostic laboratories can be found on the Regional Center websites. 
sponders and decision makers (http://www.npdn.org). Analogous to the mission of integrated research, teaching, and outreach at land grant universities that house most NPDN laboratories, NPDN's mission also is tripartite in nature, including diagnostics, education, and communications. NPDN training and education programs enhance the early detection of new pathogens and pests by providing first detectors with the information necessary to identify poten- tial new pathogens and pests, collect and submit high quality samples to NPDN diagnostic laboratories, and notify the appropriate authorities when required. NPDN has provided training to several thousand first detectors nationwide, including crop consultants and advisors, industry field personnel, public garden personnel, and cooperative extension agents. Using NPDN's resource-rich e-learning system (http://www.FirstDetector.org), more than 2,600 first

Inset 1. The consequences that result from correct and incorrect diagnoses for plant samples that are either positive or negative for a high consequence pathogen or pest can have significant economic, environmental, and social impacts.

\section{A. Consequence of True Positives}

Scenario: A confirmed positive diagnosis is rendered for a disease or pest and accurate information is communicated to those with the responsibility and authority to respond.

\section{Potential Outcomes:}

- Eradication measures are imposed and plants or plant products are destroyed (Impacts: economic, environmental, social)

- Quarantine measures are imposed and transportation and/or commerce of plants or plant products are halted (Impacts: economic, business, social)

- Mitigation measures are implemented (e.g., chemicals applied) (Impacts: economic, environmental)

\section{B. Consequence of True Negatives}

Scenario: A confirmed negative diagnosis is rendered for a disease or pest and accurate information is communicated to those with the responsibility and authority to respond.

\section{Potential Outcomes:}

- Uninfected and/or un-infested plants and plant products are transported (Impacts: none)

- Commerce proceeds uninterrupted (Impacts: none)

\section{Consequence of False Positives}

Scenario: A positive diagnosis is incorrectly rendered for a disease or pest; inaccurate information is communicated to those with the responsibility and authority to respond.

\section{Outcomes:}

- Eradication measures imposed: plants or plant products are destroyed unnecessarily (Impacts: economic, business, environmental, social)

- Quarantine measures imposed: transportation and commerce of plants or plant products halted unnecessarily (Impacts: economic, business, social)

- Mitigation measures (e.g., chemicals applied) implemented unnecessarily (Impacts: economic, environmental)

\section{Consequence of False Negatives}

Scenario: A negative diagnosis is incorrectly rendered for a disease or pest; inaccurate information is communicated to those with the responsibility and authority to respond.

\section{Outcomes:}

- Infected and/or infested plants and plant products are transported resulting in the spread of the pathogen or pest because eradication and/or quarantine of infected/infested plants and plant products were not imposed (Impacts: economic, environmental, and perhaps social)

- Establishment of pathogen or pest at the outbreak site because eradication was not attempted (Impacts: economic, environmental)

- Spread of the pathogen or pest from the outbreak site by natural (e.g., wind and rain) or human-assisted mechanisms because quarantine and/or mitigation measures were not implemented (Impacts: economic, business, environmental) 
detectors have completed over 12,000 training modules (9). NPDN diagnostic laboratories employ an array of standard and advanced technologies to ensure accurate and timely diagnoses of new and recurring pathogens and pests in natural and agricultural plant systems. NPDN's diagnosticians are highly skilled, dedicated professionals and are provided intensive training opportunities to learn advanced technologies and to obtain experience with the diagnosis of emerging pathogens and pests. NPDN's diagnosticians and administrative staff are educated to practice secure communications to ensure that information regarding new pathogens and pests is communicated efficiently and effectively to those who need that information. NPDN secure communications protocols are reviewed periodically and amended to account for changes in technologies, threats, and policies.

\section{Regulatory Responsibility and Enforcement Authority}

An effective national plant biosecurity infrastructure requires cooperation among public institutions, government agencies, commercial enterprises, and private citizens (8). These partners have distinct but complementary missions. To detect, mitigate, and manage the incursions of new pathogens and pests requires the careful coordination and integration of plant protection efforts (Fig. 3). Hence, a general understanding of the roles and responsibilities of all partners is essential. Without this understanding and cooperation, plant protection efforts can be compromised, putting in jeopardy the very plant systems and industries in need of protection. NPDN developed and implemented an outbreak scenario exercise program as a platform to facilitate cooperation through practice for plant health emergencies.

NPDN has neither regulatory responsibility nor enforcement authority for plant protection at the state or national levels. The responsibility for the enactment and implementation of plant protection regulations rests with state agencies for intrastate issues and federal agencies for interstate and international issues. The governing bodies of each state and territory have enacted plant protection laws with jurisdiction for that state or territory. State agencies (e.g., state departments of agriculture) are tasked with the implementation and enforcement of the plant protection regulations for their own state. A State Plant Regulatory Officer (SPRO) within each state provides supervision for the plant protection programs of that state. The USDA APHIS PPQ has the responsibility and enforce- ment authority for the regulation of interstate commerce and international trade of plants and plant products. For most plant trade scenarios, federal regulations supersede state regulations; hence, in most cases, USDA APHIS PPQ enforcement authority supersedes state enforcement authority. However, state and federal agencies work closely together to safeguard plant systems and plant industries. The State Plant Health Director (SPHD) in each state is an APHIS PPQ employee with state-delimited responsibilities. The SPHD works closely with the SPRO in matters of plant protection.

NPDN's role in response to the detection of new or regulated pathogens and pests is one of coordination and support (Fig. 3).

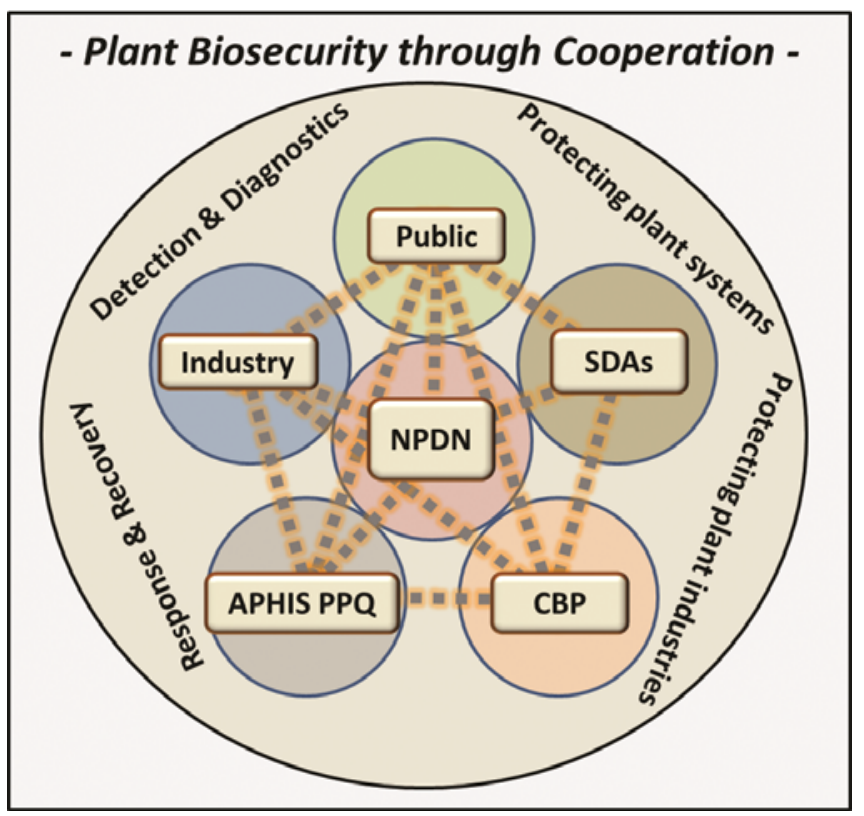

Fig. 3. The National Plant Diagnostic Network (NPDN), in concept and in practice, has become a model for cooperation among the important organizations and agencies necessary to protect our natural and agricultural plant systems. NPDN performs diagnostics and facilitates communications and interactions among its partners.

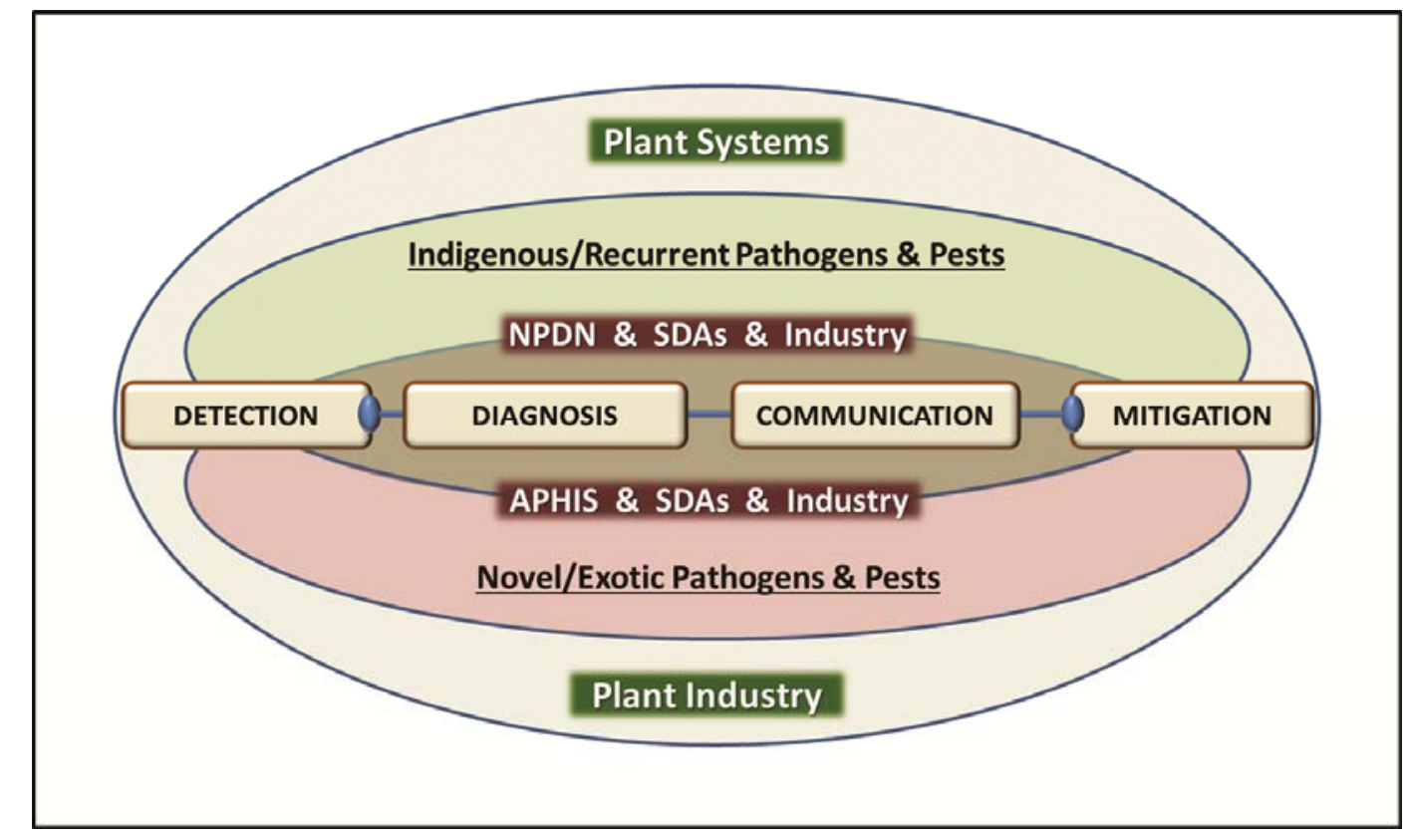

Fig. 2. The agencies and organizations that make up the U.S. national plant biosecurity infrastructure have independent but overlapping roles and responsibilities in the shared mission of protecting plant health. The National Plant Diagnostic Network (NPDN) is integral to the detection and diagnosis of recurrent and emergent indigenous plant pathogens and arthropod pests. Equally important, NPDN provides detection and triage diagnostic support to individual U.S. state departments of agriculture and to USDAAPHIS PPQ for introduced exotic plant pathogens and arthropod pests. 
Management and regulatory actions are usually based upon the result and interpretation of a diagnosis. Consequently, sample collection, processing, and diagnosis can become the rate-limiting step in the implementation of a response plan. NPDN laboratories receive and process the samples that provide the information that feeds the decision making process. State and federal agencies have the responsibility and authority to identify the expert laboratories that perform confirmatory diagnoses. Some NPDN laboratories serve as expert labs under conditional approval by APHIS to serve that role. However, the majority of NPDN labs serve in a triage capacity (13); they sort out the negatives from the presumptive positives that get forwarded to an expert laboratory for a confirmatory diagnosis.

\section{Triage and Surge Support}

NPDN is a consortium of plant diagnostic laboratories from land grant institutions, state departments of agriculture, and more recently industry. NPDN diagnostic labs perform two roles: routine diagnostics in support of the plant systems within a state and triage diagnostics associated with an outbreak or detection of new pathogens and pests $(10,13)$. The detection of a new pathogen or pest triggers a series of communications and activities designed to accurately identify the agent, delimit the area affected, and mitigate the impact of the detection and/or the new agent. Many NPDN labs are home to sophisticated diagnostic technologies and highly skilled, experienced diagnosticians. These labs perform true triage on the samples associated with an outbreak or detection; they sort out the negatives from the potential positives (Fig. 4). All potentially actionable suspect samples are then forwarded under permit within approved packaging to expert laboratories for a confirmatory diagnosis. Most often, the expert laboratory is one of the following: USDA APHIS PPQ's Center for Plant Health Science and Technology (CPHST) Beltsville Laboratory (plant samples), USDA-ARS Systematic Entomology Laboratory Communications and Taxonomic Services Unit (CTSU) (insect samples), or an NPDN Regional Hub or Expert Lab as designated by the USDA National Identification System.

One of the most important benefits of this triage system is that by screening and eliminating the negative samples, NPDN labs greatly reduce the number of potentially positive samples that need confirmation by an expert laboratory. This allows experts to

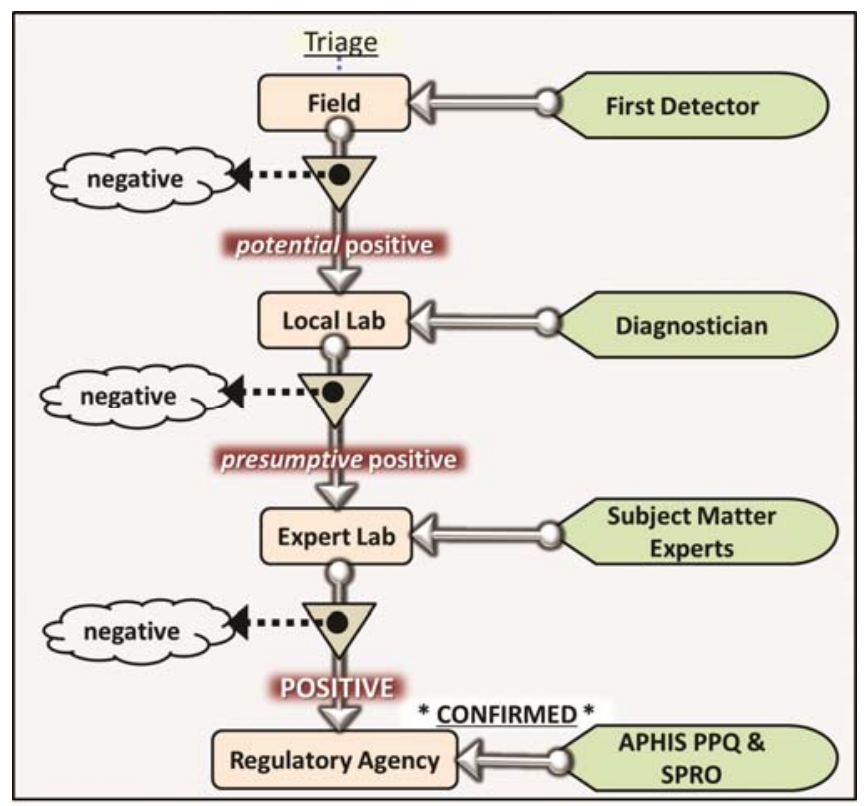

Fig. 4. By providing triage and surge support during plant disease outbreaks and arthropod pest infestations, the National Plant Diagnostic Network (NPDN) is an important partner with federal, state, and local plant protection agencies, the public and private sectors, and with the industries that support plant protection. Adapted from Stack 2010 (13). provide greater scrutiny of potentially positive samples and thus shortens the response time for confirmation and subsequent response actions. Once an NPDN diagnostician receives a sample suspected to be of regulatory concern, the proper handling of that sample, the essential communications regarding that sample, and the procedures for forwarding that sample to an expert laboratory are guided by the NPDN Chain of Custody Standard Operating Procedure (SOP) and by the experience of the diagnostician (http://www.npdn.org/exercise_ resources). The NPDN Chain of Custody SOP was developed through collaboration with USDA APHIS PPQ and is periodically reviewed and updated to ensure regulatory requirements are met and to facilitate cooperation during response to plant health emergencies.

Effective plant diagnosis relies upon a mutually dependent combination of experience and technology (10). In many cases, the diagnosis of high consequence pathogens and pests requires the sequential application of more than one technology. The national trace forward survey after the 2005 Phytophthora ramorum dissemination in nursery plants utilized a tiered approach involving morphological (culturing for chlamydospores), serological (an ELISA screen for Phytophthora species), and molecular (PCR of the ELISA positive samples) diagnostic technologies prior to confirmation of a positive detection. Every diagnostic technology has capabilities and limits with respect to specificity and sensitivity regarding the target signal or organism. It requires an experienced diagnostician or clinician to master each technology in order to understand the capabilities and limits and to successfully interpret the results generated by each technology. During the $P$. ramorum trace forward response, experience in the isolation and culturing of Phytophthora species from plant, soil, and water matrices and in the recognition of $P$. ramorum structures was essential to concluding whether a sample was positive or negative for $P$. ramorum. Similarly, experience with ELISA and/or PCR was, and still is, necessary to distinguish among real and false positives and negatives that are common with Phytophthora species.

To minimize mistakes caused by human error or equipment malfunction, it is imperative that only experienced diagnosticians conduct diagnoses for samples that may have a regulated pathogen or pest. Diagnosticians must be experienced with the approved protocols, the technologies employed, and the plant system being analyzed in order to accurately generate and interpret the results of diagnostic tests for samples with regulatory implications. This is one of the most compelling reasons for the on-going training and education program for NPDN diagnosticians, as well as for the continued development and implementation of the STAR-D Laboratory Accreditation System at NPDN labs. In partnership with USDA APHIS PPQ, NPDN is developing the STAR-D system to harmonize laboratory standards among NPDN labs, document the quality assurance and quality control practices among NPDN labs, and enhance diagnostic performance throughout the network. In addition to NPDN's internal training and education program, USDA APHIS PPQ CPHST has been a critical and essential partner in providing advanced training to NPDN diagnosticians.

The diagnostic process can take several days from initial detection to final confirmatory diagnosis. During that time, samples are in various stages of diagnosis, e.g., suspect, potentially positive, presumptive positive, confirmed positive. As different technologies and protocols are brought to bear on the diagnosis, the status of the diagnosis can change from presumptive positive to either confirmed negative or confirmed positive. The confirmatory diagnosis, whether positive or negative, can greatly impact individuals, businesses, and in some cases an entire industry. Consequently, the release of information on an as-yet-unconfirmed diagnosis can have significant unintended impacts.

\section{NPDN Policies}

NPDN communications philosophy. The philosophy of NPDN is one of collaboration and cooperation. The practice of NPDN is to establish and maintain strong working relationships with a variety of partners and stakeholders including state and federal regula- 
tory agencies, those with the responsibility and authority to respond to new plant pathogen and arthropod pest outbreaks in plant systems. This requires secure bidirectional communication pathways that facilitate the exchange of pathogen and pest outbreak information among local, state, and federal plant protection partners.

Following the detection of either an unknown or an unusual occurrence of a plant pathogen or arthropod pest, NPDN ensures effective communications through a notification protocol that informs all relevant partners that something unusual has been detected in a state or region. Specific information regarding the location of the detection and the sample collected are provided only to state and federal regulatory officials. In support of response efforts, subsequent NPDN communications may alert laboratory directors and/or diagnosticians to begin laboratory preparations for diagnostic and/or surge support. Until an expert laboratory has rendered a confirmed diagnosis, all NPDN communications are kept confidential and secure.

The primary objectives of the NPDN communications protocol are (i) to facilitate the timely communication of accurate information relevant to the detection, diagnosis, and response to plant disease outbreaks and arthropod pest infestations, and (ii) to secure information from premature disclosure under situations that may result in a regulatory action or that may adversely impact plant health management decisions. The goal of NPDN communication protocols is to facilitate the most efficient and effective response that will minimize impact and best serve the individuals and plant industries affected.

Data sharing. NPDN acquires plant diagnostic records from 50 U.S. states and all U.S. territories and archives those data in the NPDN National Data Repository at the Center for Environmental and Regulatory Information Systems (CERIS) at Purdue University. Diagnostic data records submitted to the NPDN Data Repository do not contain the identity of the individual or organization that submitted the sample nor site-specific information that would enable the identification of the individual or enterprise where the sample was collected. Only authorized NPDN and CERIS personnel are granted direct access to the NPDN Data Repository. To prevent unauthorized access to NPDN data, all NPDN and CERIS personnel, including data analysts, are trained in the practice of secure data acquisition, data handling, and data distribution in accordance with accepted information security standards. Data acquisition and storage systems for both NPDN and the Plant Diagnostic Information System (PDIS) were audited by the Center for Education and Research in Information Assurance and Security (CERIAS), an original National Security Agency center for academic excellence in information security. CERIAS provided the NPDN Regional Centers, the NPDN Data Repository, and PDIS with plans for system and information security; those plans were implemented. PDIS is a lab information management system used by more than 30 NPDN states.

Limited sharing of specific diagnostic data does occur among certain NPDN states and/or regions. Data sharing is regulated according to the NPDN Data Sharing Policy. Individual state's records within the NPDN Repository are subject to the policies of that state with respect to access to public records of this nature. Diagnostic data records that are of concern with respect to national security are exempt from disclosure through the Freedom of Information Act. NPDN's policies do not supersede the policies of individual member states.

NPDN has recognized that there are legitimate reasons to request access to diagnostic records by scientists and regulators. Requests for access to NPDN diagnostic data are reviewed by the NPDN Executive Committee and evaluated using several criteria including, the scope of the request, the integrity of the requestor and requestor's organization, whether there is a legitimate need for a scientific or regulatory purpose, etc. In all cases where the decision is made to grant access to NPDN diagnostic data, NPDN and CERIS personnel provide the requested data to the requestor. Direct access to the NPDN Data Repository is only granted to NPDN administrators, NPDN diagnosticians, and to state and federal regulatory officials.

Individual member laboratories submitting data to NPDN are responsible for the integrity and security of the data at their laboratory and their site. Access to and retention of those data are determined by the policy of each data-submitting laboratory. Individual laboratories are responsible for maintaining the integrity of the restricted access policy from their individual laboratory to the NPDN regional and national repositories. NPDN-created data products will not be distributed to entities in other countries or to the general public without the written consent of the laboratory(s) from which the data were submitted. CERIS is responsible for the integrity and security of the NPDN National Data Repository.

\section{Plant Protection through Cooperation}

Many entities comprise our national plant biosecurity infrastructure, including, federal, state, and local agencies (e.g., National Plant Board, APHIS PPQ, Department of the Interior), an array of industries (e.g., seed companies, chemical supply companies, applicators, custom harvesters, etc.), a diverse corps of first detectors (e.g., extension agents and specialists, farmers, crop advisors, consultants, etc.), and universities. These entities have distinct but overlapping missions. A continuing challenge has been to develop sound working relationships that facilitate cooperation among those who contribute to plant protection, especially during plant health emergencies. NPDN's mission is to support local, state, and national efforts for the detection and diagnosis of plant pathogens and pests of concern. It was intended to be, and has developed into, a collaborative enterprise fully integrated into our national plant biosecurity infrastructure. NPDN was embedded into the land grant university system as fully consistent with the land grant extension mission, neither adjunct to it nor distinct from it. In both philosophy and practice, NPDN is a partner in plant protection.

Because of the ever-increasing pressures on plant systems from the trade of plants and plant products, a strong plant biosecurity infrastructure is essential to sustaining the natural and agricultural plant systems upon which we depend. The plant biosecurity challenge is far too complex and the consequences too great for any one organization or sector to meet that challenge. Our success depends upon the mutual understanding and respect for the roles and responsibilities of all partners in plant protection; it depends upon collaboration and cooperation.

\section{Summary}

To address the food security needs of many nations and the economic wellbeing of most nations, plants and plant products are shipped around the world in great quantities and in short periods of time. The global movement of plants and plant products is driving the redistribution of the pathogens and pests that adversely affect plant health $(2,11,12)$. To effectively manage the potential impacts of the introduction of exotic pathogens and pests into any area requires a plant biosecurity infrastructure designed to intercept pathogens and pests at our borders and to detect and mitigate those that slip through $(5,16)$. It also requires informed biosecurity policies to facilitate international cooperation and ensure safe trade (1).

The plant biosecurity infrastructure in the United States is structurally and operationally comprised of public and private enterprises including federal and state agencies, an array of plant industries, academic institutions, crop consultants and advisors, and farmers. These partners in plant protection have distinct yet overlapping missions that, unless coordinated, could generate confusion during the response to the detection of an emerging or introduced plant pathogen or pest. NPDN is a consortium of university, state department of agriculture, and industry diagnostic laboratories with broad capabilities for the detection and diagnosis of plant pathogens and pests. NPDN has neither regulatory responsibility nor enforcement authority. NPDN labs follow standardized protocols for data acquisition and storage and practice secure communications to ensure that information of potential regulatory concern is not released inappropriately. 
Following the detection of a new pathogen or pest, there is a sense of urgency to inform those who may be affected and to quickly implement mitigation measures. However well intended that may be, the premature release of information following the detection of a new pathogen or pest can cause significant economic injury to individuals or to an entire industry. Accurate, timely, and secure communications are essential to the effective response to plant health emergencies.

\section{Acknowledgments}

We thank the many dedicated, highly skilled, and professional NPDN diagnosticians for providing such a great story to tell. The National Plant Diagnostic Network is supported, in part, by USDA National Institute of Food and Agriculture Cooperative Agreements: 2012-37620-19627, 2012-37620-19626, 201237620-19617, 2012-37620-19644, and 2012-37620-19535.

\section{Literature Cited}

1. Brasier, C. M. 2008. The biosecurity threat to the UK and global environment from international trade in plants. Plant Pathol. 57:792-808.

2. Britton, K. O. 2004. Controlling biological pollution. Pages 1-7 in: Biological Pollution, An Emerging Global Menace. K. Britton, ed. American Phytopathological Society, St. Paul, MN

3. Bullock, R., Shiel, P., Berger, P., Kaplan, D., Parra, G., Li, W., Levy, L. Keller, J., Reddy, M., Sharma, N., Dennis, M., Stack, J., Pierzynski, J., O'Mara, J., Webb, C., McKemy, J., and Palm, M. 2006. Evaluation of detection techniques to determine presence of Phytophthora ramorum in a nursery. Online. Plant Health Prog. doi:10.1094/PHP-2006-1016-01-RS

4. Cardwell, K. F., and Hoffman, W. 2008. Early Detection and Diagnosis of High Consequence Plant Pests-Intentional vs. Natural Introduction. John Wiley \& Sons, Hoboken, NJ.

5. Fletcher, J., and Stack, J. P. 2007. Agricultural biosecurity: Threats and impacts for plant pathogens. Pages 86-94 in: Institute of Medicine. 2007 Global infectious disease surveillance and detection: Assessing the challenges - finding the solutions. Workshop summary. The National Academies Press, Washington, DC.

6. Jarnevich, C. S., Graham, J. J., Newman, G. J., Crall, A. W., and

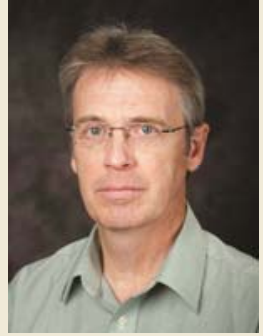

James Stack

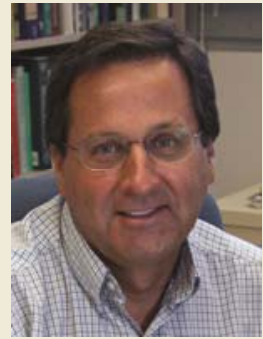

Richard Bostock

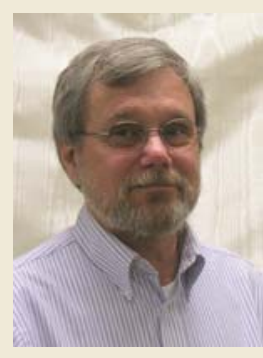

Ray

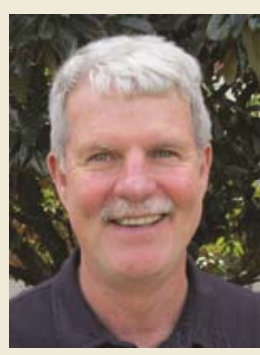

Jeff Jones

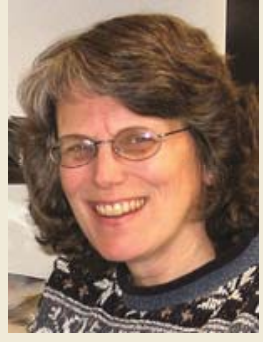

Eileen Luke

Hammerschmidt

Dr. Stack is Director of the Great Plains Diagnostic Network (GPDN) and a professor of Plant Pathology at Kansas State University. He is principal investigator of plant biosecurity projects regarding emerging diseases in Europe, the Middle East, South America, and Australia. Dr. Stack's research interests center on pathogen detection and surveillance, pathogen ecology, and epidemiology for accidental and intentional (e.g., biological control, bioterrorism) introductions of plant pathogens into natural and agricultural plant systems; his interests range from preparedness and prevention to mitigation and recovery. Of particular interest is a better understanding of the intersection of plant health, human health, and food security.

Dr. Bostock is a professor in the Department of Plant Pathology at the University of California, Davis. Dr. Bostock received his B.S. degree in biology from Rhodes College in 1974 and a Ph.D. in plant pathology at the University of Kentucky in 1981. His research and teaching interests are the biochemistry and molecular biology of plant-microbe interactions and general plant pathology. $\mathrm{He}$ and his colleagues have studied programmed cell death in response to pathogens, toxins, and elicitors, systemic signaling in resistance and susceptibility, and the influence of diverse stressors on these processes. In addition, he leads an applied research program on fungal diseases of orchard crops, with a current emphasis on thousand cankers disease of walnut and brown rot of stone fruits. $\mathrm{He}$ teaches several undergraduate and graduate courses in plant pathology, as well as a popular course entitled "Feeding the Planet" in the Science and Society program. $\mathrm{He}$ is a Fellow of the American Phytopathological Society and a Fellow of AAAS.

Dr. Hammerschmidt is professor of plant pathology and associate chairperson of the Department of Plant, Soil and Microbial Sciences at Michigan State University. He also serves as the coordinator of Diagnostic Services at MSU and is the founding and current director of the North Cen- tral Plant Diagnostic Network. He received his B.S. and M.S. degrees in biochemistry and plant pathology, respectively, from Purdue University, and his Ph.D. in plant pathology from the University of Kentucky. Dr. Hammerschmidt joined the faculty at MSU in 1980, where his research has focused on physiology and biochemistry of resistance and induced resistance in several plant systems. He was elected a Fellow of the American Phytopathological Society in 2007. He teaches introductory plant pathology for undergraduates and a graduate course in plant-pathogen interactions.

Dr. Jones is a professor in the Department of Plant Pathology at the University of Florida. He received his B.S. in botany from the University of Massachusetts and his $\mathrm{Ph} . \mathrm{D}$. in plant pathology from Virginia Tech. He has a research $(80 \%)$ and teaching $(20 \%)$ appointment with expertise in the area of bacterial diseases. In addition, Dr. Jones has served as Regional Director of the National Plant Diagnostic Network from 2009 till the present and is currently the Executive Director of the National Plant Diagnostic Network.

Ms. Luke is the director of the Center for Environmental and Regulatory Information Systems with complete management and administrative responsibilities. CERIS is a recognized center at Purdue University with 12 professional staff. For over 19 years, she has managed and led information technology development at CERIS with Information Retrieval System (NPIRS), the National Agricultural Pest Information System (NAPIS), Urban Forest Health Information Center (UFORHIC), and the National Plant Diagnostic Network (NPDN) that cover pesticide registrations, plant pest survey, urban tree inventories, and plant diagnostics, respectively. With over 25 years experience in IT development in agricultural information systems, Eileen Luke has worked with a wide variety of software and applications to provide web interface access to database systems that meet the needs of the customers. 
Stohlgren, T. J. 2007. Balancing data sharing requirements for analyses with data sensitivity 2007. Biol. Invasions (2007) 9:597-599. DOI 10.1007/s10530-006-9042-4

7. Lamour, K. H., Habera, L. F., Snover-Clift, K. L., Stack, J. P., Pierzynski, J., Hammerschmidt, R., Jacobs, J. L., Byrne, J. M., Harmon, P. F., Vitoreli, A. M., Wisler, G. C., Harmon, C. L., Levy, L. E., Zeller, K. A., Stone, C. L., Luster, D. G., and Frederick, R. D. 2006. Early detection of Asian soybean rust using PCR. Plant Health Progress: Online. Plant Health Progress doi:10.1094/PHP-2006-0524-01-RS http://www.plantmanagementnetwork. org/sub/php/research/2006/pcr/

8. Magarey, R. D., Dolezal, W. E., and Moore, T. J. 2009. Worldwide monitoring systems: The need for public and private collaboration. Pages 349-355 in: Plant Pathology in the 21st Century, vol. 1. Recent Developments in Management of Plant Diseases. U. Gisi, I. Chet, and M. L. Gullino, ed. Springer, Dordrecht, The Netherlands.

9. McCarthy, R. 2013. Leveraging NPDN's First Detector Resources. NPDN Newsl. 8(7):5-6.

10. Miller, S. A., Beed, F. D., and Harmon, C. L. 2009. Plant disease diagnostics and networks. Annu. Rev. Phytopathol. 2009.47:15-38. (www.annual reviews.org)
11. Palm, M. 1999. Mycology and world trade: A view from the front line Mycologia 91:1-12.

12. Rossman, A. 2001. A special issue on global movement of invasive plants and fungi. BioScience 51(2):93-94.

13. Stack, J. 2010. Diagnostic networks for plant biosecurity. In: Knowledge and Technology Transfer for Plant Pathology, Plant Pathology in the 21st Century 4. N. V. Hardwick and M. L. Gullino, eds. DOI 10.1007/978-14020-8934-3_6, (C) Springer Science + Business Media B.V. 2010

14. Stack, J. P., and Baldwin, W. 2008. The need for secure communications networks and global connectivity. Pages 103-109 in: Crop Biosecurity: Assuring Our Global Food Supply. M. L. Gullino, J. Fletcher, A. Gamliel, and J. P. Stack, eds. Springer, The Netherlands.

15. Stack, J., Cardwell, K., Hammerschmidt, R., Byrne, J., Loria, R., SnoverClift, K., Baldwin, W., Wisler, G., Beck, H., Bostock, R., Thomas, C., and Luke, E. 2006. The National Plant Diagnostic Network. Plant Dis. 90:128-136.

16. Stack, J. P., and Fletcher, J. 2007. Plant biosecurity infrastructure for disease surveillance and diagnostics. Pages 95-106 in: Institute of Medicine. 2007. Global infectious disease surveillance and detection: Assessing the challenges - finding the solutions. Workshop summary. The National Academies Press, Washington, DC. 